\title{
Effect of Heterologous Vaccination Strategy on Humoral Re- sponse against COVID-19 with CoronaVac plus BNT162b2: A Prospective Cohort Study
}

\author{
Hakan Demirhindi ${ }^{*}$, Burak Mete ${ }^{2}$, Ferdi Tanir ${ }^{3}$, Ertan Kara ${ }^{4}$, Filiz Kibar ${ }^{5}$, Salih Cetiner ${ }^{6}$, Aslihan Candevir ${ }^{7}$, \\ Şükriye Ece Akti ${ }^{8}$
}

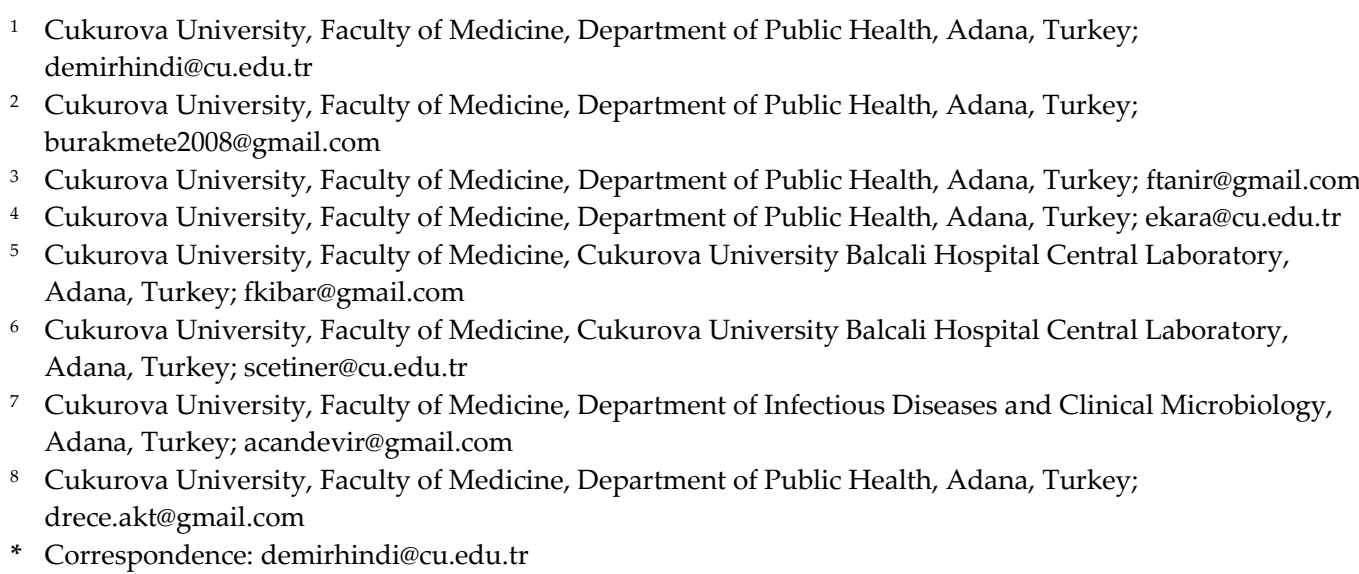

\begin{abstract}
This study aimed to evaluate the mixed and homogeneous application of the inactivated SARS-CoV-2 vaccine CoronaVac (CV) and the mRNA vaccine BNT162b2 (BNT). This prospective cohort study included 235 health care workers, who had received two prime shots with CoronaVac. They were divided into three cohorts after the third month: Cohort-I (CV/CV); Cohort-II $(\mathrm{CV} / \mathrm{CV} / \mathrm{CV})$ and Cohort-III (CV/CV/BNT). Anti-S-RBD-IgG and total anti-spike/anti-nucleocapsidIgG antibody concentrations were examined in vaccinated health workers at the 1st, 3rd and 6th months following the second dose of the vaccination. The mean age of 235 health care workers who participated in the project was $39.51 \pm 10.39$ (min-max: 22-64). At the end of the 6th month, no antibodies were detected in $16.7 \%$ of Cohort-I participants, and anti-S-RDB IgG levels showed a decrease of $60 \%$ compared to the levels of the $3 \mathrm{rd}$ month. The antibody concentrations of the 6 th month were found to have increased by an average of 5.13 times compared to the 3rd-month levels in the Cohort-II and 20.4 times in Cohort-III. The heterologous vaccination strategy "CoronaVac and BNT162b2 regimen" is able to induce a stronger immunity and it will help remove inequalities in the developing world where CoronaVac was the initial prime.
\end{abstract}

Keywords: COVID-19 vaccines, seroconversion, inactivated SARS-CoV2 vaccine, BNT162 Vaccine; COVID-19 vaccine booster shot, heterologous vaccination, mixed vaccination, vaccination strategy

\section{Introduction}

The SARS-CoV-2 is well known as a new member of the Betacoronavirus (CoVs) family, which is the leading cause of severe acute respiratory syndrome (SARS) [1-3]. The vaccine research rally reached more than 50 vaccine candidates against COVID-19 at the end of 2020. There were included different platforms such as nucleic acid, live attenuated virus, protein subunit, and viral vector vaccines [4]. In a short time, several large clinical trials have demonstrated the clinical efficacy and safety of certain COVID-19 vaccines. Each of these platforms has advantages, as well as disadvantages. As an example, nucleic acid-based vaccines such as DNA vaccines or mRNA vaccines are easy to design, 
but they may not be immunogenic. Moreover, evidence suggests that the mRNA vaccines are more unstable in comparison to the other types of vaccines. Viral vector-based vaccines show higher safety and are more immunogenic, while they probably have low efficiency due to the preexisting immunity to the vector [5]. From all designed vaccines, only a few have entered the clinical trials. Although none of these vaccines has completed clinical trials, there are still many attempts in advance to develop such a vaccine [6]. Currently, the correlation between the vaccine-elicited immune response and the protection from SARS-CoV-2 infection remains unclear, and it is not known how changes in immunity could be reflected in clinical outcomes. Doria-Rose et al. showed that mRNA1273-elicited antibodies could persist for six months after the second dose in a phase- 1 trial comprising 333 healthy participants [7]. But the durability of protection induced by COVID-19 vaccination remains to be determined. On this basis, Khoury et al. analysed seven vaccines and a convalescent cohort to design a predictive model for revealing the relationship between the observed protection and in vitro neutralization levels from COVID-19. They showed that neutralising antibody levels are highly predictive of immune protection. For achieving a 50\% protection the neutralisation level was estimated to be approximately $20.2 \%$ of the average convalescent level (95\% CI, 14-28) and $3 \%$ (95\% CI, $0.7-13)$ for $50 \%$ protection from severe infection, and that the decay of neutralizing titres in vaccinated subjects over the first 3-4 months after vaccination was, at least, as rapid as the decay observed in the convalescent subjects. Furthermore, modeling the neutralization titres decay over the first 250 days after immunisation showed that a significant loss in protection from SARS-CoV-2 infection would while protection from severe disease would be largely preserved [8].

Heterologous prime-boost vaccination, which defines mixing different vaccine types, is a strategy originated in the 1990s to be used against HIV infection by aiming to induce both T-cell and B-cell immunity. The 30-years cumulative result of these vaccine platforms, which included DNA, mRNA and viral vectors, is what rendered the development of COVID-19 vaccines so quickly possible (reminding that this does not include DNA vaccines). Mixing vaccines is based on the idea of presenting the antigen to the immune system in a different way. This approach is particularly important for viral vector vaccines against COVID-19 like Sputnik V, Johnson\&Johnson, CanSino Biologics and AstraZeneca vaccines that use a replication-deficient adenovirus as a vector. But the immune system may attack the vector virus itself rendering the vaccine ineffective. To avoid this, it is accepted that third shots would be shifted to mRNA or protein-based vaccines following the prime series with vector vaccines. The main aim is effective and long-lasting protection [9]. There are several studies comparing immune responses and any side effects of the combination of the prime shots with one type and a booster shot of another type like Com-COV (1-2-3) or COV-Boost studies in the United Kingdom. They comprise AstraZeneca, Pfizer\&BioNTech, Moderna, and Novavax vaccines [10,11]. The next generation of vaccines are expected to be against several coronavirus variants and therefore a broader immunity, longevity of response and a good safety profile is the major aim of all vaccine studies and a broad collective immunity in case of mixing vaccines [9].

We aimed in this study to evaluate whether the mixed application of the inactivated vaccine and the mRNA vaccine BNT162b2 had a superiority in antibody response and safety compared to homologous vaccine application.

\section{Materials and Methods}

\subsection{Study design and participants}


This was a prospective cohort study and constituted the third step of a vaccine efficacy and safety project. It was carried out at Cukurova University (Adana, Turkey) in September 2021 and included health care workers who had been vaccinated with two doses of the inactivated SARS-CoV-2 vaccine in the context of a public vaccination program by the Turkish Ministry of Health (TR-MoH). The health care workers constituted the priority group in the vaccination target population. The incidence of adverse events in the vaccine cohort was determined to be $19 \%$ after the administration of $3 \mu \mathrm{g}$ (corresponding to $600 \mathrm{SU}$ ) of the inactivated SARS-CoV-2 vaccine during Phase-2 clinical trials [12]. The minimum sample size was calculated as 220 participants by assuming type- 1 error as 0.05 and type- 2 error as 0.20 considering reported adverse events rates. The participants were randomly selected from a list of 3,000 health care workers. Two substitution lists were also prepared by randomisation. A total number of 282 health care workers participated in the first step of the study performed at the end of the first month after vaccination. This number decreased to 272 in the second step of the study performed at the end of the third month after the second dose of the vaccine when 10 participants resigned from the study of their own free will. The third step of the study included 235 participants and the calculations were based on the results of these participants.

Blood samples were taken after the participants had been informed about the study and had signed an informed consent form. They answered a questionnaire form about sociodemographic characteristics, natural COVID-19 infection history, vaccination and adverse events.

\subsection{Vaccine information}

\subsubsection{Inactivated SARS-CoV-2 vaccine by Sinovac (CoronaVacTM)}

The vaccine administered to health care workers by the TR-MoH is "inactivated SARSCoV-2 vaccine (CoronaVacTM)", with aluminium hydroxide, developed by Sinovac Biotech Ltd., Life Sciences Lab., China. The vaccine was administered intramuscularly in the deltoid region of the upper arm with a dosage of $3 \mu \mathrm{g} / 0.5 \mathrm{ml}$. The two doses were administered 28 days apart. The vaccines were transferred, stored and administered following cold-chain principles already in use by, and therefore familiar to health institutions performing vaccinations.

\subsubsection{BNT162b2 mRNA vaccine by Pfizer \& BioNTech (Comirnaty®)}

The vaccine BNT162b2 (Comirnaty ${ }^{\circledR}$ ) produced by BioNTech Manufacturing $\mathrm{GmbH}$, Germany is a nucleoside-modified messenger-RNA (mRNA) encapsulated in lipid nanoparticles (LNP), which enable the delivery of the RNA into host cells to allow expression of the SARS-CoV-2 spike (S) antigen. The vaccine elicits both neutralising antibody and cellular immune responses to the $S$ antigen, which may contribute to protection against COVID-19 disease. The mRNA of the vaccine is a highly purified single-stranded, 5 'capped mRNA produced using a cell-free in-vitro transcription from the corresponding DNA templates, encoding the S protein of SARS-CoV-2. This vaccine is a white to offwhite frozen suspension provided as a multiple-dose vial and must be diluted before use. One vial $(0.45 \mathrm{~mL})$ contains 6 doses of $0.3 \mathrm{~mL}$ after dilution. One dose $(0.3 \mathrm{~mL})$ contains 30 micrograms of COVID-19 mRNA vaccine (embedded in lipid nanoparticles) [13].

\subsubsection{Mixed Vaccine administration}

In June 2021 the Turkish Republic MoH declared the introduction of an additional shot of a third dose to health care workers and elderly who had previously received two 
doses of CoronaVac and to the individuals who wished to receive it due to some international travel requirements. All individuals were given the right to choose between CoronaVac and the BNT162b2 vaccine at their free will.

\subsection{Immunogenicity assessments}

The project aimed to measure anti-SARS-CoV-2 S-RBD (anti-S-RBD) immunoglobulin G (IgG), total anti-spike and anti-nucleocapsid immunoglobulin IgG antibody concentrations in the same individuals repetitively, i.e. at the end of the first month (first step of the study), in the third month (second step) and in the sixth month (third step) after the administration of the second dose of CoronaVac. As the TR-MoH introduced the third dose (booster) in the vaccination schedule in June 2021, the evaluation of the same antibodies following this booster dose was also included in this presented third step of the study, performed at the end of the sixth month. As the individuals were given the right to choose, the third (booster) dose consisted of either CoronaVac or BNT162b2. Therefore, all participants were divided into three cohorts:

-Cohort-I (CV/CV) included those who received only two doses of CoronaVac,

-Cohort-II (CV/CV/CV) included those who received three doses of CoronaVac and

-Cohort-III (CV/CV/BNT) included those who received two doses (prime) of CoronaVac followed by the third dose (booster) with BNT162b2.

About $5 \mathrm{~mL}$ of blood samples were collected into biochemistry tubes with vacuum gel. The sera were extracted by centrifugation at $3000 \mathrm{gv}$ for 10 minutes and kept at $2-8^{\circ} \mathrm{C}$ for 1-3 days. Test calibrators and controls were performed first. After the control results were observed to be within the expected ranges, the samples were tested by trained experts in the accredited (by the Joint Commission International (JCI) since 2006) Central Laboratory of Cukurova University Balcali Hospital, Adana, Turkey.

The blood samples stored at $2-8^{\circ} \mathrm{C}$ were brought to room temperature on the day the tests were performed and examined collectively after all participants were sampled. The participants were not informed about their antibody concentration since the start of the study (in a single-blind format) to avoid a possible effect on the declaration of clinical complaints or outcomes as they would be followed repetitively for six months. The test method used for the qualitative detection and differentiation of IgG antibodies (indirect method) to SARS-CoV-2 in human serum and serum in separating gel tubes is a chemiluminescence immunoassay (CLIA) reaction based on the measurement of light signals by a photomultiplier as relative light units (RLUs) proportional to the concentration of antibodies present in the sample [14,15]. The test is only for use according to the Food and Drug Administration's Emergency Use Authorization [16]. The SARS-CoV-2 S-RBD IgG test is also an indirect CLIA and has a high correlation with VNT50 titres ( $\mathrm{R}=0.712)$, where VNT stands for "Virus Neutralization Test" which is a gold standard for quantifying the titre of neutralising antibodies (nAbs) for a virus [16]. The results are expressed in absorbance units $(\mathrm{AU} / \mathrm{mL})$ and reported to the end-user as "Reactive (i.e. a result $\geq 1.00$ $\mathrm{AU} / \mathrm{mL}$ )" or "Non-Reactive (i.e. a result $<1.00 \mathrm{AU} / \mathrm{mL}$ )" [14].

\subsection{Statistical analyses}

Data were examined using the SPSS 22 statistical analyses package (2013, IBM, New York, U.S.A). Following normality testing (Kolmogorov-Smirnov), data were analysed by Freidman and Kruskal Wallis tests. A value of $\mathrm{p}<0.05$ was considered significant. 


\section{Results}

The mean age of 235 health care workers who participated in the third step of our study was 39.51 \pm 10.39 (between 22 and 64). All participants received two doses of CoronaVac (CV) one month apart at the start of the study. At the end of the sixth month following the second dose; $21.3 \%$ of the participants reported to have received only two doses of CoronaVac in the Cohort-I (CV/CV), $7.2 \%$ to have received three doses of CoronaVac in the Cohort-II $(\mathrm{CV} / \mathrm{CV} / \mathrm{CV})$, and $71.5 \%$ to have received two doses of CoronaVac followed by the third dose with BNT162b2 (BNT) vaccine in the Cohort-III (CV/CV/BNT).

Some participants reported a COVID-19 infection history before the first dose in all three cohorts $(6,5$ and 26, respectively). But those who reported the infection after the second dose were observed in the $\mathrm{CV} / \mathrm{CV}$ cohort (2 people) and the $\mathrm{CV} / \mathrm{CV} / \mathrm{BNT}$ cohort (7 people), the latter being observed between the second and third doses. There were not any infection history between the second and third doses in the $\mathrm{CV} / \mathrm{CV} / \mathrm{CV}$ cohort.

When the participants who had received the third dose were considered, the mean of days passed between the second and third dose administrations was 148.3 days for the $\mathrm{CV} / \mathrm{CV} / \mathrm{CV}$ cohort and 142.8 days for the $\mathrm{CV} / \mathrm{CV} / \mathrm{BNT}$ cohort. Eight participants were not included in the analyses, because the interval between the third shot and the blood sampling for our study was shorter than 14 days. This interval was found to range between 18 and 71 days (Table 1 ).

Table 1. Sociodemographic characteristics of the participants according to vaccine cohorts

\section{Vaccine cohorts}

$\mathrm{n}(\%)$ or mean \pm standard deviation (min-max)

\begin{tabular}{lrrr}
\hline Characteristics & Cohort-I & Cohort-II & Cohort-III \\
\hline Sex & & & \\
Male & $24(24.5)$ & $7(7.1)$ & $67(68.4)$ \\
Female & $26(19.0)$ & $10(7.3)$ & $101(73.7)$ \\
\hline Chronic disease present & & & \\
Yes & $11(18.0)$ & $4(6.6)$ & $46(75.4)$ \\
No & $38(22.0)$ & $13(7.5)$ & $122(70.5)$ \\
\hline History of COVID-19 infection & & & $12(6.3)$ \\
$\quad$ Never & $42(22.0)$ & $5(13.5)$ & $26(70.3)$ \\
Before the 1st dose & $6(16.2)$ & $0(0)$ & $7(71.4)$ \\
$\quad$ After the 2nd dose & $2(28.6)$ & $39.29 \pm 8.18$ & $40.67 \pm 10.94$ \\
\hline Age & $35.66 \pm 8.13$ & $148.35 \pm 15.32$ & $142.85 \pm 10.48$ \\
\hline Interval between & & $(136-180)$ & $(136-186)$ \\
$2^{\text {nd }}$ and 3rd doses (days) & & $39.52 \pm 10.50$ & $45.92 \pm 12.47$ \\
\hline Interval between & & $(18-50)$ & $(18-71)$ \\
$2^{\text {nd }}$ dose and blood sampling (days) & & \\
\hline
\end{tabular}


At the end of the sixth month, no antibodies could be detected in $16.7 \%$ of the individuals in the CV/CV cohort and the level of anti-S-RDB IgG was found to be significantly decreased by a mean of $60 \%$ compared to the third month. The anti-S-RDB IgG was found detectable in all individuals in the CV/CV/BNT cohort. The level of antibodies in the sixth month was found to have increased by an average of 5.13 times in the $\mathrm{CV} / \mathrm{CV} / \mathrm{CV}$ cohort and 20.4 times in the CV/CV/BNT cohort. Anti-S-RBD IgG levels were found to be significantly higher in the $\mathrm{CV} / \mathrm{CV} / \mathrm{BNT}$ group than in the $\mathrm{CV} / \mathrm{CV} / \mathrm{CV}$ group or $\mathrm{CV} / \mathrm{CV}$ group. In other words, the participants who received the BNT vaccine as the third dose presented higher IgG levels than all other cohorts (Table 2).

Table 2. Anti-S-RBD IgG levels according to months and vaccine cohorts

\begin{tabular}{|c|c|c|c|c|c|c|c|c|}
\hline & & \multicolumn{3}{|c|}{ Anti-S-RBD IgG (AU/ml) } & \multicolumn{3}{|c|}{ Antibody proportions } & \multirow{2}{*}{$\frac{\text { Immunity }}{N / R^{c}}$} \\
\hline Vaccine cohorts & & $\begin{array}{c}\text { 1st } \\
\text { month }\end{array}$ & $\begin{array}{c}\text { 3rd } \\
\text { month }\end{array}$ & 6th month & $\mathrm{p}$ & 6th/1st ${ }^{a}$ & 6 th $/ 3 \mathrm{rd}^{\mathrm{b}}$ & \\
\hline Cohort-I & Mean & 44.38 & 23.47 & 11,14 & & & & \\
\hline \multirow[t]{3}{*}{$\mathrm{n}=42$} & S.D. & 49.84 & 40.51 & 24.97 & $<0.001$ & 0.64 & 0.40 & $16.7 / 83.3$ \\
\hline & Median & 27.57 & 7.53 & 2.66 & & & & \\
\hline & Range & 203.73 & 229.62 & 119.14 & & & & \\
\hline Cohort-II & Mean & 46.61 & 15.02 & 76.18 & & & & \\
\hline \multirow[t]{3}{*}{$\mathrm{n}=12$} & S.D. & 55.53 & 15.65 & 53.28 & 0.003 & 3.53 & 5.13 & $0 / 100.0$ \\
\hline & Median & 13.99 & 8.61 & 85.99 & & & & \\
\hline & Range & 158.16 & 55.46 & 139.06 & & & & \\
\hline Cohort-III & Mean & 42.34 & 17.59 & 136.82 & & & & \\
\hline \multirow[t]{3}{*}{$\mathrm{n}=137$} & S.D. & 37.73 & 27.17 & 11.00 & $<0.001$ & 17.00 & 20.44 & $0 / 100.0$ \\
\hline & Median & 29.63 & 9.21 & 139.20 & & & & \\
\hline & Range & 146.10 & 214.71 & 116.40 & & & & \\
\hline
\end{tabular}

The individuals with a history of COVID-19 before or after the vaccinations were excluded from the analyses

${ }^{a} \mathrm{IgG}$ level changes as a proportion of $6^{\text {th }}$-month to $1^{\text {st-month levels }}$

${ }^{\mathrm{b}} \mathrm{IgG}$ level changes as a proportion of $6^{\text {th }}$-month to $3^{\text {rd }}$-month levels

${ }^{\mathrm{c}} \mathrm{N}$ : Non-Reactive, R: Reactive

When the total anti-spike/anti-nucleocapsid IgG antibody level in the sixth month was compared to that of the third month, it was found to be undetectable in $71.4 \%$ in the $\mathrm{CV} / \mathrm{CV}$ cohort with a decrease of $91 \%$; to have increased by an average of 3.5 times in the $\mathrm{CV} / \mathrm{CV} / \mathrm{CV}$ cohort, and to have decreased by $87 \%$ in the $\mathrm{CV} / \mathrm{CV} / \mathrm{BNT}$ cohort (Table 3 ).

Table 3. Total anti-spike/anti-nucleocapsid IgG levels according to months and vaccine cohorts

\begin{tabular}{llrrrrrrr}
\hline & \multicolumn{4}{c}{ Total anti-spike/anti-nucleocapsid IgG } & \multicolumn{2}{c}{ Antibody proportions } & Immunity \\
\hline Vaccine cohort & & 1st month & 3rd month & 6th month & p & 6/1 & 6/3 & N/R (\%) \\
\hline Cohort-I & Mean & 30.26 & 22.72 & 1.91 & & & & \\
$\mathrm{n}=42$ & S.D. & 38.68 & 45.32 & 3.77 & $<\mathbf{0 . 0 0 1}$ & 0.30 & 0.09 & $71.4 / 28.6$ \\
& Median & 11.98 & 3.39 & 0.054 & & & &
\end{tabular}




\begin{tabular}{|c|c|c|c|c|c|c|c|c|}
\hline & Range & 143.02 & 251.08 & 15.80 & & & & \\
\hline Cohort-II & Mean & 12.81 & 9.92 & 22.01 & & & & \\
\hline \multirow[t]{3}{*}{$\mathrm{n}=12$} & S.D. & 11.36 & 12.82 & 28.64 & 0.035 & 4.15 & 3.58 & $16.7 / 83.3$ \\
\hline & Median & 10.60 & 2.85 & 9.42 & & & & \\
\hline & Range & 35.05 & 36.69 & 89.43 & & & & \\
\hline Cohort-III & Mean & 27.74 & 19.01 & 1.82 & & & & \\
\hline \multirow[t]{3}{*}{$n=137$} & S.D. & 44.08 & 49.10 & 5.64 & $<0.001$ & 0.10 & 0.13 & $75.2 / 24.8$ \\
\hline & Median & 10.88 & 3.82 & 0.062 & & & & \\
\hline & Range & 367.68 & 407.32 & 40.72 & & & & \\
\hline
\end{tabular}

The individuals with a history of COVID-19 before or after the vaccinations were excluded from the analyses ${ }^{\text {a }} \mathrm{IgG}$ level changes as a proportion of $6^{\text {th }}$-month to $1^{\text {st-month levels }}$

${ }^{b}$ IgG level changes as a proportion of $6^{\text {th }}$-month to $3^{\text {rd }}$-month levels

The distribution of anti-S-RBD IgG levels according to months and vaccine cohorts are presented in Figure 1.

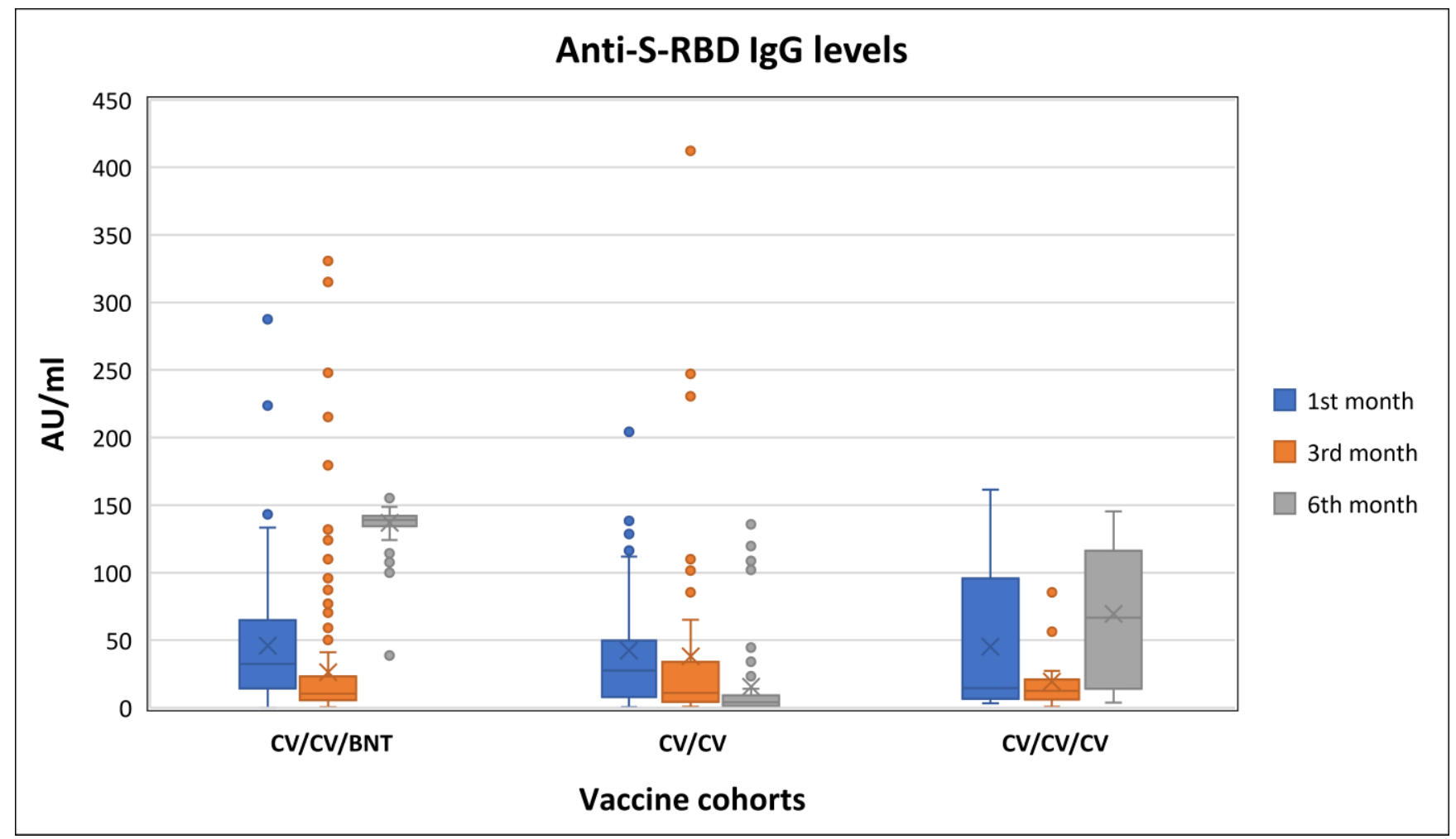

Figure 1. The distribution of anti-S-RBD IgG levels according to months and vaccine cohorts

When the anti-S-RBD IgG levels were compared between the vaccine cohorts, it was found that they had a statistically significant decrease of $51 \%$ among participants without any history of COVID-19 infection in the CV/CV cohort. However, among the participants who had the infection before the first dose or after the second dose in the same $\mathrm{CV} / \mathrm{CV}$ cohort, the reduction of antibody levels at the sixth month was not statistically significant. 
In the $\mathrm{CV} / \mathrm{CV} / \mathrm{CV}$ cohort, the antibody levels were found to have increased 6.4 times in those who had COVID-19 history. But the increase was 1.5 times among those who had the infection before the start of the vaccinations.

In the CV/CV/BNT cohort, the level of antibodies increased 23 times among participants without COVID-19 history, but 2.3 times who got infected before the vaccinations, and 8.8 times in those who got infected after the second dose, but before the third dose of the vaccine. The decrease was observed to be at a slower rate in individuals with a positive history of natural COVID-19 infection (Table 4).

Table 4. Anti-S-RBD IgG levels according to months, vaccine cohorts and COVID-19 history

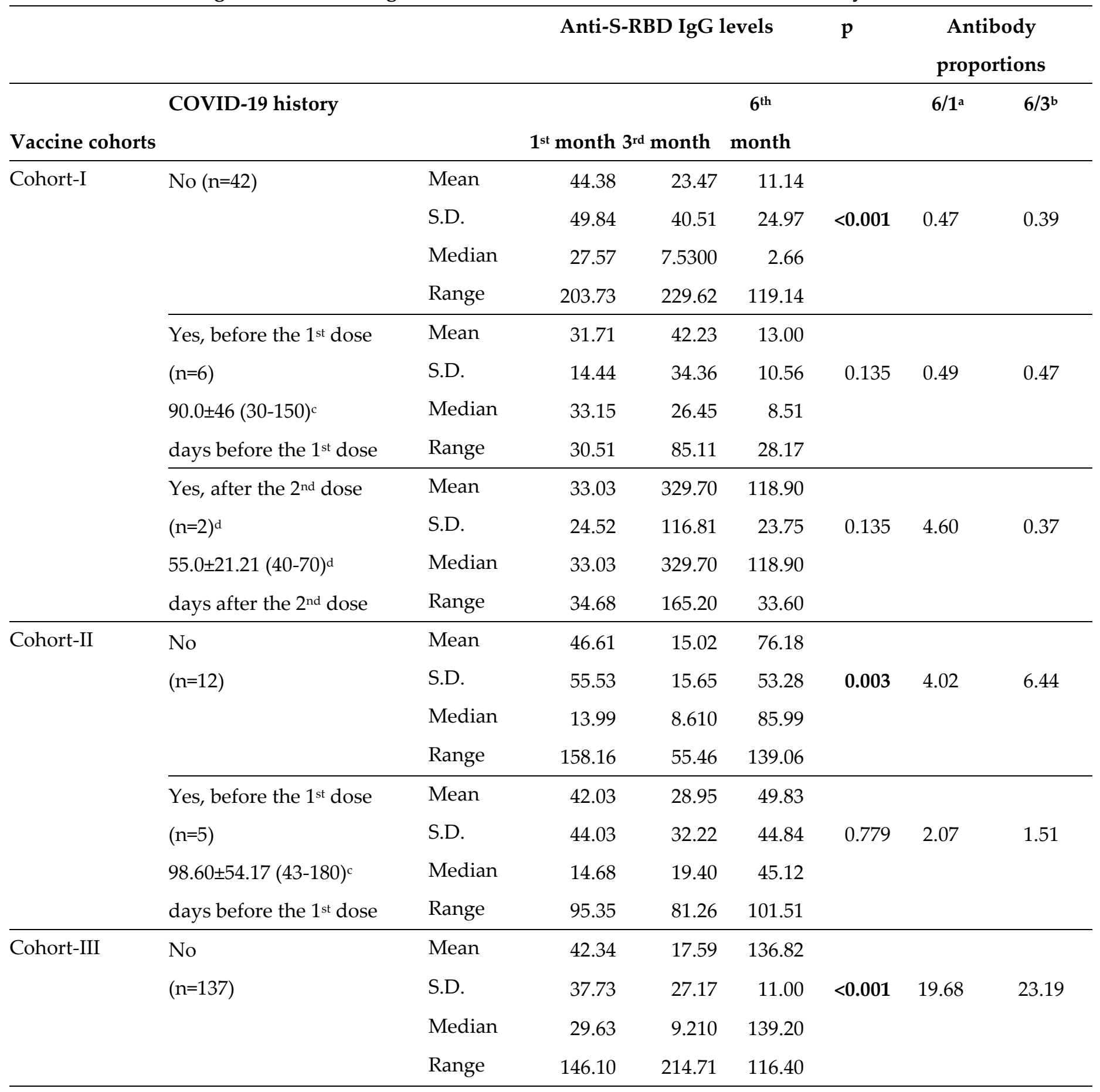




\begin{tabular}{|c|c|c|c|c|c|c|c|}
\hline Yes, before the vaccinations & Mean & 66.28 & 42.41 & 137.70 & \multirow{4}{*}{$<0.001$} & \multirow{4}{*}{5.33} & \multirow{4}{*}{8.89} \\
\hline$(n=26)$ & S.D. & 69.09 & 45.71 & 4.74 & & & \\
\hline $130.2 \pm 91.31(30-330)^{c}$ & Median & 38.48 & 22.02 & 137.75 & & & \\
\hline days before the 1st dose & Range & 280.32 & 175.18 & 18.300 & & & \\
\hline \multicolumn{2}{|c|}{ Yes, between the $2^{\text {nd }}$ and $3^{\text {rdMean }}$} & 39.91 & 188.00 & 134.22 & & & \\
\hline dose & S.D. & 16.40 & 153.94 & 19.28 & 0.247 & 4.02 & 2.94 \\
\hline$(\mathrm{n}=5)$ & Median & 33.41 & 248.00 & 142.70 & & & \\
\hline $88.0 \pm 51.67(60-180)^{\mathrm{d}}$ & Range & 38.95 & 319.73 & 45.70 & & & \\
\hline
\end{tabular}

For all of the participants, the history of natural COVID-19 infection dates either before the first dose or between the second and third dose of vaccinations $\mathrm{c}, \mathrm{d}$

a IgG level changes as a proportion of $6^{\text {th }}$-month to $1^{\text {st }}$-month levels

${ }^{\mathrm{b}} \mathrm{IgG}$ level changes as a proportion of $6^{\text {th }}$-month to $3^{\text {rd }}$-month levels

c Time interval of COVID-19 infection before the vaccinations in days (mean \pm standard deviation, min-max)

$\mathrm{d}$ Time interval of COVID-19 infection between the second and third dose of the vaccinations in days (mean \pm standard deviation, minimum-maximum)

The anti-S-RBD IgG antibody levels were found to have decreased in all age groups of the $\mathrm{CV} / \mathrm{CV}$ cohort, while, in contrast, to have increased in all age groups of the $\mathrm{CV} / \mathrm{CV} / \mathrm{CV}$ cohort and the $\mathrm{CV} / \mathrm{CV} / \mathrm{BNT}$ cohort. The increase was higher in the $\mathrm{CV} / \mathrm{CV} / \mathrm{BNT}$ cohort than the $\mathrm{CV} / \mathrm{CV} / \mathrm{CV}$ cohort (Table 5).

Table 5. Anti-S-RBD IgG levels in vaccine cohorts according to the age groups

\begin{tabular}{|c|c|c|c|c|c|c|c|c|}
\hline & & \multicolumn{5}{|c|}{ Anti-S-RBD IgG levels } & \multicolumn{2}{|c|}{$\begin{array}{l}\text { Antibody } \\
\text { proportions }\end{array}$} \\
\hline \multirow[b]{2}{*}{ Vaccine cohorts } & & & & & $6^{\text {th }}$ & $\mathbf{p}$ & $6 / 1^{a}$ & $6 / 3^{b}$ \\
\hline & \multicolumn{2}{|l|}{ Age (years) } & \multicolumn{2}{|c|}{$1^{\text {st }}$ month $3^{\text {rd }}$ month } & month & & & \\
\hline \multirow[t]{11}{*}{ Cohort-I } & $20-29$ & Mean & 42.91 & 16.11 & 4.82 & & & \\
\hline & $(n=12)$ & S.D. & 42.30 & 22.92 & 6.59 & $<0.001$ & 0.31 & 0.34 \\
\hline & & Median & 35.61 & 8.08 & 2.15 & & & \\
\hline & & Range & 129.93 & 84.31 & 24.01 & & & \\
\hline & $30-39$ & Mean & 67.04 & 39.44 & 22.10 & & & \\
\hline & $(\mathrm{n}=17)$ & S.D. & 61.91 & 57.35 & 36.72 & $<0.001$ & 0.27 & 0.47 \\
\hline & & Median & 48.64 & 25.63 & 6.26 & & & \\
\hline & & Range & 202.24 & 229.62 & 119.14 & & & \\
\hline & $40-49$ & Mean & 15.17 & 9.77 & 2.74 & & & \\
\hline & $(\mathrm{n}=11)$ & S.D. & 11.96 & 11.37 & 2.47 & $<0.001$ & 1.04 & 0.35 \\
\hline & & Median & 9.97 & 4.67 & 1.91 & & & \\
\hline
\end{tabular}




\begin{tabular}{|c|c|c|c|c|c|c|c|c|}
\hline & & Range & 34.04 & 38.40 & 8.21 & & & \\
\hline & 50 and & Mean & 21.36 & 7.21 & 2.19 & & & \\
\hline & $(\mathrm{n}=2)$ & S.D. & 14.70 & 3.84 & 1.07 & 0.135 & 0.11 & 0.30 \\
\hline & & Median & 21.36 & 7.21 & 2.19 & & & \\
\hline & & Range & 20.80 & 5.44 & 1.52 & & & \\
\hline Cohort-II & $20-29$ & Mean & 98.43 & 32.31 & 94.41 & & & \\
\hline & $(\mathrm{n}=2)$ & S.D. & 89.32 & 33.96 & 53.71 & 0.223 & 1.20 & 4.57 \\
\hline & & Median & 98.43 & 32.31 & 94.41 & & & \\
\hline & & Range & 126.33 & 48.04 & 75.97 & & & \\
\hline & $30-39$ & Mean & 28.70 & 10.52 & 99.12 & & & \\
\hline & $(n=5)$ & S.D. & 42.42 & 8.422 & 52.96 & 0.018 & 7.02 & 10.65 \\
\hline & & Median & 12.89 & 9.99 & 105.30 & & & \\
\hline & & Range & 99.98 & 20.39 & 135.69 & & & \\
\hline & $40-49$ & Mean & 102.25 & 20.42 & 94.14 & & & \\
\hline & $(n=2)$ & S.D. & 18.30 & 9.71 & 36.56 & 0.223 & 0.96 & 5.67 \\
\hline & & Median & 102.25 & 20.42 & 94.14 & & & \\
\hline & & Range & 25.89 & 13.74 & 51.71 & & & \\
\hline & 50 and & Mean & 4.80 & 5.90 & 13.81 & & & \\
\hline & $(n=3)$ & S.D. & 1.46 & 1.09 & 9.43 & 0.264 & 2.94 & 2.59 \\
\hline & & Median & 4.62 & 5.86 & 10.56 & & & \\
\hline & & Range & 2.92 & 2.18 & 18.00 & & & \\
\hline Cohort-III & $20-29$ & Mean & 49.34 & 22.95 & 138.94 & & & \\
\hline & $(n=26)$ & S.D. & 40.50 & 29.62 & 4.81 & $<0.001$ & 6.19 & 15.47 \\
\hline & & Median & 34.14 & 10.39 & 139.60 & & & \\
\hline & & Range & 140.81 & 121.32 & 17.00 & & & \\
\hline & $30-39$ & Mean & 44.10 & 14.74 & 137.29 & & & \\
\hline & $(n=30)$ & S.D. & 36.72 & 17.40 & 7.14 & $<0.001$ & 7.64 & 18.98 \\
\hline & & Median & 29.95 & 9.08 & 138.10 & & & \\
\hline & & Range & 130.97 & 85.35 & 34.90 & & & \\
\hline & $40-49$ & Mean & 45.97 & 13.72 & 137.92 & & & \\
\hline & $(n=43)$ & S.D. & 40.64 & 16.13 & 7.09 & $<0.001$ & 8.92 & 23.34 \\
\hline & & Median & 25.56 & 9.07 & 139.90 & & & \\
\hline & & Range & 127.45 & 96.56 & 40.90 & & & \\
\hline & 50 and & Mean & 32.05 & 20.56 & 133.76 & & & \\
\hline & $(\mathrm{n}=38)$ & S.D. & 32.12 & 39.18 & 17.82 & $<0.001$ & 50.61 & 30.49 \\
\hline & & Median & 17.88 & 7.93 & 138.15 & & & \\
\hline
\end{tabular}




Range $\quad 143.20 \quad 214.71 \quad 109.80$

a IgG level changes as a proportion of $6^{\text {th }}$-month to $1^{\text {st }}$-month levels

${ }^{\mathrm{b}} \mathrm{IgG}$ level changes as a proportion of $6^{\text {th }}$-month to $3^{\text {rd }}$-month levels

The adverse events were found to be more frequent in participants who received the mRNA vaccine as the third dose of the vaccination (Table 6).

Table 6 . The adverse events after the third dose of the vaccination

\begin{tabular}{|c|c|c|}
\hline \multirow[b]{2}{*}{ Adverse events } & \multicolumn{2}{|c|}{ Vaccine administered as the third dose [n(line \%)] } \\
\hline & Inactivated vaccine (n:17) & mRNA vaccine $(\mathrm{n}: 168)$ \\
\hline Total & $3(2.5)$ & $116(97.5)$ \\
\hline Rash at the injection site & $0(0.0)$ & $13(100.0)$ \\
\hline Pain at the injection site & $1(0.9)$ & $113(99.1)$ \\
\hline Swelling at the injection site & $1(5.0)$ & $19(95.0)$ \\
\hline Itching at the injection site & - & $9(100.0)$ \\
\hline Hypoesthesia at the injection site & - & $2(100.0)$ \\
\hline Induration at the injection site & - & $15(100.0)$ \\
\hline Numbness in the vaccinated arm & - & $18(100.0)$ \\
\hline Weakness & $2(3.5)$ & $55(96.5)$ \\
\hline Fatigue & - & $48(100.0)$ \\
\hline Fever & - & $19(100.0)$ \\
\hline Shake & - & $8(100.0)$ \\
\hline Chest pain & - & $5(100.0)$ \\
\hline Diarrhoea & - & $2(100.0)$ \\
\hline Nausea & - & $7(100.0)$ \\
\hline Vomiting & - & $2(100.0)$ \\
\hline Headache & $1(3.4)$ & $28(96.6)$ \\
\hline Dizziness & $1(3.7)$ & $26(96.3)$ \\
\hline Vertigo & - & $10(100.0)$ \\
\hline Muscle pain & - & $30(100.0)$ \\
\hline Back pain & - & $21(100.0)$ \\
\hline Joint pain & - & $23(100.0)$ \\
\hline Cough & - & $2(100.0)$ \\
\hline Sore throat & - & $2(100.0)$ \\
\hline Dyspnea & - & $3(100.0)$ \\
\hline Papule & - & - \\
\hline Abdominal pain & - & $1(100.0)$ \\
\hline
\end{tabular}




\begin{tabular}{lcr}
\hline Loss of appetite & - & $6(100.0)$ \\
Palpitation & - & $6(100.0)$ \\
Anosmia & - & $1(100.0)$ \\
Loss of taste & - & - \\
Rash in the skin/mucosa & - & - \\
Numbness in the tongue & - & - \\
Syncope & - & - \\
Increased blood pressure & - & - \\
Decreased blood pressure & - & - \\
Allergic reaction/urticaria & - & - \\
Anaphylaxis/anaphylactoid reaction & - & $3(100.0)$ \\
Neurological complications & - & - \\
Lymphadenopathy & - &
\end{tabular}

\section{Discussion}

\section{Discussion}

Ensuring an effective and durable immune response is very important in reaching the herd immunity threshold essential for ending the pandemic. In the face of limited vaccine supply, a mixed-vaccine strategy was proposed to protect as many people as possible. On this basis, a 13-month study was launched by Shaw et al. on February 4, 2021, to determine the effects of using different vaccines for each dose in different testing arms: (1) the ChAdOx1 nCov-19 vaccine (AZD1222) by AstraZeneca for the first dose followed by the BNT162b2 for the second dose 28 days later; (2) the AZD1222 for the first dose, followed by the BNT162b2 for the second dose 12 weeks later; (3) the BNT162b2 vaccine for the first dose, followed by the AZD1222 vaccine for the second 28 days later; and (4) the BNT162b2 vaccine for the first dose, followed by the AZD1222 for the second dose 12 weeks later. The initial report showed that there was an increase in systemic reactogenicity after the boost dose, as reported by participants in heterologous vaccine schedules in comparison to homologous vaccine schedules [17]. Another trial is being performed to examine the safety and immunogenicity of combining the AZD1222 and rAd26-S (GamCOVID-Vac; Sputnik V) vaccines, which are both adenovirus vector vaccines. One group received AZD1222 on day-1 followed by rAd26-S on day-29 and the second group received the same vaccines in the reverse order. Preliminary Phase-2 results of the study revealed a 4-fold or greater increase in neutralising antibody levels compared to the baseline [18]. In a study conducted in Spain by Borobia et al. between April 24 and April $30,2021,676$ individuals were randomized (450 in the intervention and 226 in the control group) at five sites in Spain, among them 663 (441 and 222, respectively) completed the study up to day 14 (mean age $44,56.5 \%$ female). In the intervention group, geometric mean titres (GMT) of IgG-RBD were found to increase from $71.46 \mathrm{BAU} / \mathrm{mL}(95 \% \mathrm{CI}$, 59.84-85.33) at baseline to 7756.68 (95\% CI, 7371.53-8161.96) at day $14(\mathrm{p}<0.0001)$. IgG against trimeric spike-protein increased from 98.4 (95\% CI, 85.69-112.99) to 3684.87 (95\% CI, 3429.87-3958.83). All of the participants exhibited neutralising antibodies 14 days after BNT162b2 administration, in comparison to $34.1 \%$ at enrolment. A 4-fold increase in cellular immune response was also observed. Reactions were predominantly mild 
$(68.3 \%)$ or moderate $(29.9 \%)$, and consisted of injection site pain $(88.2 \%)$, induration $(35.5 \%)$, headache $(44.4 \%)$ and myalgia (43.3\%). No serious adverse events were reported. BNT162b2 given as a second dose in individuals primely vaccinated with ChAdOx1-S induced a robust immune response with an acceptable and manageable reactogenicity profile [19]. In a study at Charité-Universitätsmedizin Berlin in Germany including 340 health care workers immunised between December 27, 2020 and May 21, 2021, it was aimed to assess reactogenicity and immunogenicity of heterologous primeboost immunisations of ChAdOx1 followed by BNT compared to homologous BNT/BNT immunisation. Heterologous ChAdOx/BNT booster vaccination was overall well-tolerated and reactogenicity was largely comparable to homologous BNT/BNT vaccination. Systemic reactions were most frequent after prime immunisation with ChAdOx $(86 \%$, 95\%CI, 79-91), and less frequent after homologous BNT/BNT (65\%, 95\%CI, 56-72), or heterologous ChAdOx/BNT booster vaccination (48\%, 95\%CI, 36 -59$)$. Serum antibody responses and $\mathrm{T}$ cell reactivity were found to strongly increase after both homologous and heterologous boost, and immunogenicity was overall robust, and comparable between both regimens in this cohort, with slightly increased S1-IgG avidity and T-cell responses following heterologous booster immunisation. Evidence of rare thrombotic events associated with $\mathrm{ChAdOx}$ has led to the recommendation of a heterologous booster with mRNA vaccines for certain age groups in several European countries, despite a lack of robust safety and immunogenicity data for this vaccine regimen. This interim analysis provided evidence that the currently recommended heterologous ChA$\mathrm{dOx} / \mathrm{BNT}$ immunisation regimen with 10-12 week vaccine intervals was well tolerated and slightly more immunogenic compared to homologous BNT/BNT vaccination with three weeks vaccine intervals. Heterologous prime-boost immunisation for COVID-19 was concluded to be generally applicable to optimise logistics, improve immunogenicity and mitigate potential intermittent supply shortages for individual vaccines [20]. In three recent studies, researchers reported that following one dose of ChAdOx with a dose of the BNT had produced strong immune responses, as measured by blood tests. Two of the studies even suggested that the mixed vaccine response would be at least as protective as two doses of the BNT vaccine, one of the most effective COVID-19 vaccines. Only a few of the potential vaccine combinations were tested. But if mixing vaccines proves safe and effective, it could speed the effort to protect billions of people [21].

In our study, heterologous use of inactivated SARS-CoV-2 vaccine (CoronaVacTM) and mRNA vaccine BNT162b2 (Comirnaty $\left.{ }^{\circledR}\right)$ was compared with homologous vaccination in terms of immunity and adverse reactions. We found that heterologous administration of CoronaVac and BNT162b2 vaccines produced a stronger immune response than the homologous regimen. At the end of the sixth month, antibodies could not be detected in $16.7 \%$ of the individuals who received two doses of the inactivated vaccine $(\mathrm{CV} / \mathrm{CV})$, and the level of anti-S-RDB IgG decreased by $60 \%$ on average compared to the thirdmonth levels. After the third dose of CoronaVac the antibody level in the sixth month increased by an average of 5.13 times compared to the third month, and 20.4 times in those who received the BNT162b2 vaccine. In terms of adverse events, it was observed that $97.5 \%$ of the side effects were in the heterologous vaccine group, while $2.5 \%$ of them were in the homologous vaccine group. Similarly, Pérez-Then et al. reported that heterologous CoronaVac prime followed by BNT162b2 booster regimen induced elevated virus-specific antibody levels and potent neutralization activity against the ancestral virus and Delta variant, resembling the titers obtained after two doses of mRNA vaccines. They also pointed out that $\mathrm{CV} / \mathrm{CV} / \mathrm{BNT}$ resulted in a 1.4-fold increase in neutralization activity against Omicron, compared to BNT/BNT regimen [22].

\section{Conclusions}


In conclusion, our study revealed that heterologous administration of inactivated SARSCoV-2 (CoronaVac) prime followed by BNT162b2 (Comirnaty) booster regimen produced a stronger immune response than the homologous inactivated vaccine regimen, but more adverse events were observed. About $17 \%$ of people who received only two doses of the CoronaVac vaccine lacked antibodies. One of the important results of the study is that a booster dose is needed at the end of the sixth month. Mixed (heterologous) vaccination with the inactivated SARS-CoV-2 vaccine and BNT162b2 mRNA vaccine, seems to be more effective in providing stronger immunogenicity. Mixed vaccine strategies need more evidence. If mixed vaccine strategies prove to be effective, vaccination programs will be more flexible; thereby reducing the negative impact of supply chain disruptions. Our findings have also immediate implications for multiple countries that previously used the CoronaVac regimen.

\section{Author Contributions:}

Conceptualization, H.D., B.M., F.T., E.K., F.K., S.Ç., A.C and Ş.E.A.; methodology, H.D., B.M., F.T. and E.K.; validation, F.K. and S.Ç.; formal analysis, B.M.; investigation, H.D., B.M., F.T. and E.K.; resources, H.D., B.M., F.T., E.K., F.K., S.Ç., A.C and Ş.E.A.; data curation, H.D., B.M., F.T., E.K., F.K., S.Ç., and Ş.E.A.; writing - original draft preparation, H.D. and B.M.; writing - review and editing, H.D., B.M., F.T. and E.K.; supervision, H.D. and B.M; project administration, H.D.; funding acquisition, H.D. All authors have read and agreed to the published version of the manuscript.

Funding: The project was supported by THE SCIENTIFIC RESEARCH PROJECTS OFFICE OF THE UNIVERSITY OF ÇUKUROVA, TURKEY (Grant number: TSA-2021-13642)

Institutional Review Board Statement: The study was approved by (1)The Scientific Research Platform of the Turkish Ministry of Health and (2) the Scientific Ethics Committee for Non-interventional Clinical Studies of the Cukurova University Faculty of Medicine (Adana, Turkey) (Decree no. 35, Meeting No. 108, 12.02.2021).

Informed Consent Statement: The official invitation letters with the list of the randomly selected participants and substitutions were sent to the department headships in order to let them invite the selected staff to participate in the study. Informed written consents were obtained from all participants after required acknowledgement for participation in the study and the publishing of this paper.

Data Availability Statement: The data presented in this study are available on request from the corresponding author. The data are not publicly available due to personal data protection regulations.

Conflicts of Interest: The authors declare no conflict of interest. The funders had no role in the design of the study; in the collection, analyses, or interpretation of data; in the writing of the manuscript, or in the decision to publish the results". 


\section{References}

1. Huang, C.; Wang, Y.; Li, X.; Ren, L.; Zhao, J.; Hu, Y.; Zhang, L.; Fan, G.; Xu, J.; Gu, X.; et al. Clinical features of patients infected with 2019 novel coronavirus in Wuhan, China. Lancet 2020, 395, doi:10.1016/S0140-6736(20)30183-5.

2. Cyranoski, D. Did pangolins spread the China coronavirus to people? Nature 2020, doi:10.1038/d41586-020-00364-2.

3. Chan, J.F.W.; Yuan, S.; Kok, K.H.; To, K.K.W.; Chu, H.; Yang, J.; Xing, F.; Liu, J.; Yip, C.C.Y.; Poon, R.W.S.; et al. A familial cluster of pneumonia associated with the 2019 novel coronavirus indicating person-to-person transmission: a study of a family cluster. Lancet 2020, 395, doi:10.1016/S0140-6736(20)30154-9.

4. World Health Organization Draft Landscape of COVID-19 candidate vaccines Available online: https://www.who.int/docs/default-source/a-future-for-children/novel-coronavirus_landscape_covid19.pdf?sfvrsn=4d8bd201_1 (accessed on Feb 5, 2022).

5. Shang, W.; Yang, Y.; Rao, Y.; Rao, X. The outbreak of SARS-CoV-2 pneumonia calls for viral vaccines. npj Vaccines 2020, 5, 18, doi:10.1038/s41541-020-0170-0.

6. Soleimanpour, S.; Yaghoubi, A. COVID-19 vaccine: where are we now and where should we go? Expert Rev. Vaccines 2021, 20.

7. Doria-Rose, N.; Suthar, M.S.; Makowski, M.; O’Connell, S.; McDermott, A.B.; Flach, B.; Ledgerwood, J.E.; Mascola, J.R.; Graham, B.S.; Lin, B.C.; et al. Antibody Persistence through 6 Months after the Second Dose of mRNA-1273 Vaccine for Covid-19. N. Engl. J. Med. 2021, 384, doi:10.1056/nejmc2103916.

8. Khoury, D.S.; Cromer, D.; Reynaldi, A.; Schlub, T.E.; Wheatley, A.K.; Juno, J.A.; Subbarao, K.; Kent, S.J.; Triccas, J.A.; Davenport, M.P. Neutralizing antibody levels are highly predictive of immune protection from symptomatic SARS-CoV-2 infection. Nat. Med. 2021, 27, 1205-1211, doi:10.1038/s41591-021-01377-8.

9. Ekin, A. Five things to know about: Mixing and matching coronavirus vaccines Available online: https://ec.europa.eu/research-and-innovation/en/horizon-magazine/five-things-know-about-mixingand-matching-coronavirus-vaccines (accessed on Feb 5, 2022).

10. Oxford Vaccine Group COM-COV Comparing COVID-19 vaccine schedule combinations Available online: https://comcovstudy.org.uk/home (accessed on Feb 5, 2022).

11. Southampton University COV-BOOST Evaluating COVID-19 vaccine boosters Available online: https://www.covboost.org.uk/home (accessed on Feb 5, 2022).

12. Zhang, Y.; Zeng, G.; Pan, H.; Li, C.; Hu, Y.; Chu, K.; Han, W.; Chen, Z.; Tang, R.; Yin, W.; et al. Safety, tolerability, and immunogenicity of an inactivated SARS-CoV-2 vaccine in healthy adults aged 18-59 years: a randomised, double-blind, placebo-controlled, phase 1/2 clinical trial. Lancet Infect. Dis. 2021, 21, 181-192, doi:10.1016/S1473-3099(20)30843-4. 


\section{BioNTech PRODUCT MONOGRAPH INCLUDING PATIENT MEDICATION INFORMATION} COMIRNATY(R) COVID-19 Vaccine, mRNA Suspension for Intramuscular Injection Multiple Dose Vial (after dilution each vial contains $6 \uparrow$ doses of $0.3 \mathrm{~mL}$ ) Active Immunizing Agent.

14. Diagnostic Snibe MAGLUMI 2019-nCoV IgM/IgG Immunoassay 2020.

15. Snibe Diagnostic Fact Sheet for Healthcare Providers - MAGLUMI 2019-nCoV IgM/IgG 2020.

16. Food and Drug Administration (U.S.A). EUA Authorized Serology Test Performance, in Coronavirus Disease 2019 (COVID-19) Emergency Use Authorizations for Medical Devices, F.D.A. 2021.

17. Shaw, R.H.; Stuart, A.; Greenland, M.; Liu, X.; Van-Tam, J.S.N.; Snape, M.D. Heterologous primeboost COVID-19 vaccination: initial reactogenicity data. Lancet 2021, 397.

18. Mikhail Samsonov (R-Pharm) Study in Adults of AZD1222 and rAd26-S Administered as Heterologous Prime-Boost Regimen for the Prevention of COVID-19 Available online: https://clinicaltrials.gov/ct2/show/NCT04684446?term=NCT04684446\&draw=2\&rank=1 (accessed on Feb 5, 2022).

19. Borobia, A.M.; Carcas, A.J.; Pérez Olmeda, M.T.; Castaño, L.; Jesús Bertrán, M.; García-Pérez, J.; Campins, M.; Portolés, A.; Gonzalez-Perez, M.; García Morales, M.T.; et al. Reactogenicity and Immunogenicity of BNT162b2 in Subjects Having Received a First Dose of ChAdOx1s: Initial Results of a Randomised, Adaptive, Phase 2 Trial (CombiVacS). SSRN Electron. J. 2021, doi:10.2139/ssrn.3854768.

20. Hillus, D.; Schwarz, T.; Tober-Lau, P.; Hastor, H.; Thibeault, C.; Kasper, S.; Helbig, E.T.; Lippert, L.J.; Tscheak, P.; Schmidt, M.L.; et al. Safety, reactogenicity, and immunogenicity of homologous and heterologous prime-boost immunisation with ChAdOx1-nCoV19 and BNT162b2: a prospective cohort study. medRxiv 2021, 2021.05.19.21257334, doi:10.1101/2021.05.19.21257334.

21. Vogel, G. Mixing COVID-19 vaccines appears to boost immune responses Available online: https://www.science.org/content/article/mixing-covid-19-vaccines-appears-boost-immune-responses (accessed on Feb 5, 2022).

22. Pérez-Then, E.; Lucas, C.; Monteiro, V.S.; Miric, M.; Brache, V.; Cochon, L.; Vogels, C.B.F.; Malik, A.A.; De la Cruz, E.; Jorge, A.; et al. Neutralizing antibodies against the SARS-CoV-2 Delta and Omicron variants following heterologous CoronaVac plus BNT162b2 booster vaccination. Nat. Med. 2022, doi:10.1038/s41591-022-01705-6. 